\title{
Clinical Pharmacokinetics
}

\section{COMMENT ON: Effect of Age-Related Factors on the Pharmacokinetics of Lamotrigine and Potential Implications for Dose Optimisation in Epilepsy Patients \\ --Manuscript Draft--}

\begin{tabular}{|c|c|}
\hline Manuscript Number: & CPKA-D-18-00106 \\
\hline \multicolumn{2}{|l|}{ Funding Information: } \\
\hline \multicolumn{2}{|l|}{$\begin{array}{l}\text { Corresponding Author Secondary } \\
\text { Information: }\end{array}$} \\
\hline Corresponding Author's Institution: & University College London \\
\hline \multicolumn{2}{|l|}{$\begin{array}{l}\text { Corresponding Author's Secondary } \\
\text { Institution: }\end{array}$} \\
\hline \multicolumn{2}{|l|}{ First Author Secondary Information: } \\
\hline \multirow[t]{6}{*}{ Order of Authors: } & Joseph F. Standing, Ph.D. \\
\hline & Brian Anderson \\
\hline & Stefanie Hennig \\
\hline & Nick Holford \\
\hline & Trevor Johnson \\
\hline & Catherijne Knibbe \\
\hline Suggested Reviewers: & \\
\hline
\end{tabular}




\section{COMMENT ON: Effect of Age-Related Factors on the Pharmacokinetics of Lamotrigine and Potential Implications for Dose Optimisation in Epilepsy Patients}

Joseph F Standing, 1, Brian J Anderson, 2, Stefanie Hennig, 3, Nick H Holford, 4, Trevor Johnston,

5, Catherijne AJ Knibbe, 6, Dagan O Lonsdale, 7, Amin Rostami-Hodjegan 8

1 Great Ormond Street Institute of Child Health, University College London, London, UK

2 Department of Anaesthesia, University of Auckland, New Zealand

3 School of Pharmacy, The University of Queensland, Australia

4 Department of Pharmacology and Clinical Pharmacology, University of Auckland, New Zealand

5 Certara UK, Sheffield, UK

6 Division of Systems Biomedicine and Pharmacology, Leiden Academic Centre for Drug

Research, University of Leiden, Netherlands

7 Department of Clinical Pharmacology, Institute for Infection and Immunity, St George's University of London, UK

8 Centre for Applied Pharmacokinetic Research (CAPKR), University of Manchester, UK

Address for correspondence: Dr Joseph Standing Room 661, UCL Great Ormond Street Institute for Child Health, London WC1N 1EH Email: j.standing@ucl.ac.uk Tel: 02079052370 Fax: 0207 9052882

Number of tables: 0 Number of figures: 1

Keywords: lamotrigine; pharmacokinetics; NONMEM; paediatrics

Dear Editor,

We read with interest the recent paper detailing the pharmacokinetics of lamotrigine by van Dijkman et all and would like to congratulate the authors for compiling such a comprehensive 
dataset, which they have used to evaluate apparent clearance (CL/F) changes from young infants

to elderly adults. In particular these results are important in patients aged younger than 2 years for whom the drug is currently unlicensed. We note that an extensive erratum ${ }^{2}$ has attempted to correct the interpretation of the proposed dosing guidelines, although recommendations for patients with different co-medications would have been useful. Before considering dose guidelines derived from the model however, we feel there is a more fundamental question on the underlying assumptions in model that warrants further discussion; namely the proposed function to describe changes in $\mathrm{CL} / \mathrm{F}$ with age.

In Figure 1 we have plotted the change in predicted values of CL/F with post natal age (PNA) as reported by the authors, using a continuous function to predict typical weight for age ${ }^{3}$. Here it can be seen that CL/F peaks at a post-menstrual age (PMA) of approximately 110 weeks (PNA of 1.3 years), then declines, and does not reach the same rate again until approximately 280 weeks PMA (PNA of 4.6 years). Between us we have extensive experience of modelling pharmacokinetic studies over large age ranges ${ }^{4-8}$, analysed how clearance in general changes for thousands of hypothetical drugs (e.g. see Calvier et $a^{\beta}$ in this journal), and systematically reviewed clearance maturation functions in children ${ }^{10,11}$. The authors describe an extremely rare (possibly the first) case of decreasing $\mathrm{CL} / \mathrm{F}$ with increasing age in infants and young children. Since $\mathrm{CL} / \mathrm{F}$ determines steady-state concentration, and in this case maintenance dose, it is important that this change in $\mathrm{CL} / \mathrm{F}$ with age is further explored. There are several possible explanations:

Firstly, the arbitrary step function used to describe decreasing $C L / F$ with age above 65 years may be causing an under-estimation of the true young adult value, and hence the dematuration function the authors report is merely a result of this under-estimate exacerbated by limited data in children aged 4-12 years. A more granular breakdown of goodness-of-fit to age groups of less than 1, 1-2, 3-4 year olds and 12-30 year olds would show whether model fit was consistent amongst each age group.

A second possibility is that bioavailability $(F)$ is for some reason lower in infants taking the immediate release formulation, consequently making $\mathrm{CL} / \mathrm{F}$ seem high. This does seem unlikely 
since immediate and extended release formulations have been reported to be bioequivalent ${ }^{12}$, and lamotrigine is generally well absorbed, but changes in bioavailability with age cannot be ruled out.

A third possibility, and one we think most likely, is that drug-drug interactions are causing confounding given that different age groups had different co-medication frequencies. Re-fitting the model to data only in patients not taking carbamazepine, phenytoin or valproic acid and reevaluating maturation parameters would determine this. Such an analysis would give confidence that the relative contribution of maturation and drug-drug interactions are correctly captured by the model. Further insight may also be achieved through PBPK analysis which may predict how CL/F could change with both age and in the presence of drug-drug interactions.

This finding of decreasing CL/F with increasing age in infants and young children is a novel unexpected result, and further exploration to confirm whether it is a real phenomenon, and if so why it happens with lamotrigine, is required.

\section{Figure Legend}

Figure 1: Plot of lamotrigine CL/F versus age estimated by van Dijkman et a/

\section{References}

1. Dijkman, S. C. van, Jager, N. C. B. de, Rauwe, W. M., Danhof, M. \& Della Pasqua, O. Effect of Age-Related Factors on the Pharmacokinetics of Lamotrigine and Potential Implications for Maintenance Dose Optimisation in Future Clinical Trials. Clin Pharmacokinet (2018).

2. Dijkman, S. C. van, Jager, N. C. B. de, Rauwe, W. M., Danhof, M. \& Della Pasqua, O.

Correction to: Effect of Age-Related Factors on the Pharmacokinetics of Lamotrigine and Potential Implications for Maintenance Dose Optimisation in Future Clinical Trials. Clin Pharmacokinet (2018).

3. Sumpter, A. L. \& Holford, N. H. Predicting weight using postmenstrual age-neonates to adults. Paediatr Anaesth 21, 309-315 (2011). 
4. Wang, C. et al. A bodyweight-dependent allometric exponent for scaling clearance across the 38 human life-span. Pharm. Res. 29, 1570-1581 (2012).

5. Lonsdale, D. O. et al. Standard beta-lactam doses fail to achieve PK/PD targets in critically ill patients of all ages, with children particularly at risk: results from the ABDose study. 28th ECCMID meeting, Madrid, Spain 00808, (2018).

6. Hennig, S., Standing, J. F., Staatz, C. E. \& Thomson, A. H. Population pharmacokinetics of tobramycin in patients with and without cystic fibrosis. Clin Pharmacokinet 52, 289-301 (2013).

7. McCune, J. S. et al. Busulfan in infant to adult hematopoietic cell transplant recipients: a population pharmacokinetic model for initial and Bayesian dose personalization. Clin. Cancer Res. 20, 754-763 (2014).

8. Holford, N. H., Ma, S. C. \& Anderson, B. J. Prediction of morphine dose in humans. Paediatr Anaesth 22, 209-222 (2012).

9. Calvier, E. A. et al. Allometric Scaling of Clearance in Paediatric Patients: When Does the Magic of 0.75 Fade? Clin Pharmacokinet 56, 273-285 (2017).

10. Holford, N., Heo, Y. A. \& Anderson, B. A pharmacokinetic standard for babies and adults. $J$ Pharm Sci 102, 2941-2952 (2013).

11. Germovsek, E., Barker, C. I., Sharland, M. \& Standing, J. F. Scaling clearance in paediatric pharmacokinetics: All models are wrong, which are useful? Br J Clin Pharmacol 83, 777-790 (2017).

12. Polepally, A. R. et al. Steady-state pharmacokinetics and bioavailability of immediate-release and extended-release formulations of lamotrigine in elderly epilepsy patients: Use of stable isotope methodology. J Clin Pharmacol 55, 1101-1108 (2015). 
